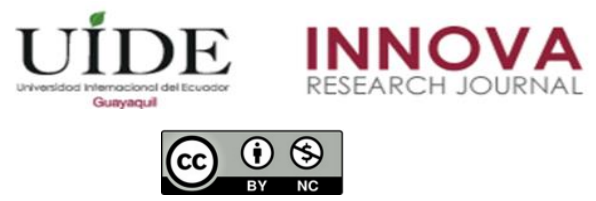

INNOVA Research Journal, ISSN 2477-9024

(Septiembre-Diciembre 2020). Vol. 5, No.3 pp. 152-163

DOI: https://doi.org/10.33890/innova.v5.n3.2020.1469

URL: http://revistas.uide.edu.ec/index.php/innova/index

Correo: innova@uide.edu.ec

\title{
Uso de las Tecnologías de Información y Comunicación en el aula: una aplicación del Teorema de Bayes
}

\section{Use of Information and Communication Technologies in the classroom: an application of Bayes' Theorem}

Fernando José Zambrano Farías

https://orcid.org/0000-0001-6384-3353

Universidad Internacional del Ecuador, Ecuador

Universidad de Guayaquil, Ecuador

Robin Xavier Martínez Mayorga

https://orcid.org/0000-0002-8193-6251

Universidad de Guayaquil, Ecuador

María Estefanía Sánchez Pacheco

https://orcid.org/0000-0002-2469-9018

Universidad Internacional de La Rioja, España

José Fernando Zambrano García

https://orcid.org/0000-0001-6685-6897

Universidad de Guayaquil, Ecuador

Autor para correspondencia: fezambranofa@uide.edu.ec; robin.martinezm@ug.edu.ec; estefania_sanchez_outlook.com; jose.zambranog@ug.edu.ec

Fecha de recepción: 17 de julio del 2020 - Fecha de aceptación: 15 de agosto del 2020

\section{Resumen}

La implementación y aplicación de las Tecnologías de la Información y Comunicación en la educación enfrenta aún muchos desafíos; consideradas como herramientas que dan soporte al proceso enseñanza-aprendizaje presencial, ofrecen excelentes escenarios para que su efectividad y eficacia mejoren la calidad de la educación superior. El objetivo de esta investigación es analizar cómo la aplicación de estas herramientas tecnológicas dentro del aula de clases mejora en el rendimiento académico promedio de los estudiantes que cursaron la materia de Matemáticas Financieras de la carrera de Administración de Empresas de la Universidad de Guayaquil en el periodo académico 2018 - 2019 ciclos I y II, para esto se hizo un análisis descriptivo de dos muestras independientes y una aplicación de la regla de Bayes. Los resultados que arrojó esta investigación concluyen que los estudiantes que acceden y aplican herramientas tecnológicas dentro del aula tienen un mejor rendimiento académico que aquellos estudiantes que no lo aplican. Palabras claves: TIC; educación; rendimiento académico; regla de Bayes; análisis descriptivo 


\begin{abstract}
The implementation and application of Information and Communication Technologies in education still face many challenges; considered as tools that support the face-to-face in teachinglearning process, they offer excellent scenarios for their effectiveness and efficiency to improve the quality of higher education. The objective of this research is to analyze how the application of these technological tools in the classroom improves the average academic performance of the students who studied the subject of Financial Mathematics in the Business Administration course at the University of Guayaquil in the period academic 2018 - 2019 cycles I and II, for this, a descriptive analysis of two independent samples and an application of the Bayes' rule were made. The results of this research conclude that students who access and apply technological tools in the classroom have better academic performance than those students who do not apply it.
\end{abstract}

Key words: ICT; education; academic performance; Bayes' rule; descriptive analysis

\title{
Introducción
}

En la actualidad, el rol que toma el conocimiento y la forma de transmitirlo dentro de la sociedad, según Sánchez et al., (2009, p. 180), genera una implantación diversificada de las tecnologías de la información y comunicación (TIC) que regirán el futuro de los sistemas económicos, las interacciones humanas y evidentemente los procesos y circunstancias del aprendizaje y de la educación.

Es tanta la importancia de las TIC que el Banco Mundial define a las tecnologías de información y comunicación como «uno de los cuatro pilares fundamentales que miden el grado de avance en el marco de la economía del conocimiento a aquellos países que las poseen» (apud Romaní, 2011, p. 297).

Es necesario acudir a la definición de TIC para lograr un mejor entendimiento de las mismas. Se definen como el conjunto de recursos o herramientas utilizados para administrar, procesar y compartir información por medio de soportes tecnológicos entre los que destacan computadoras, teléfonos móviles, televisores, entre otros.

En esta misma línea Ibáñez y García (2009, p. 21) definen las TIC como «un conjunto de herramientas electrónicas utilizadas para la recolección, almacenamiento, tratamiento, difusión y transmisión de la información representada de forma variada». Melo (2011, p. 220) afirma que las tecnologías de información y comunicación son «un conjunto de herramientas, equipos, programas informáticos, aplicaciones, redes y medios, que permiten la compilación, procesamiento, almacenamiento, transmisión como voz, datos, textos, ideas e imágenes» (apud Barreto et al., 2017, p. 15).

En el mundo actual, la integración de las TIC hacia los diferentes sectores de la comunidad productiva tiene un lugar trascendental. En el contexto educativo han llegado a ser prioridad en todos sus niveles debido a que contribuyen al acceso universal de la educación, la equidad en la construcción, aprendizaje de calidad y ayudan al perfeccionamiento profesional y académico de los docentes (Alderete y Formichella, 2016, p. 90). Sin embargo, la incorporación de éstas y su correcta aplicación dentro del sistema educativo ha sufrido dificultades debido, entre muchos factores, a la falta de preparación del cuerpo docente y a la ausencia de una política 
institucional que permita aprovecha de manera más eficiente la utilización de las TIC. Al respecto, García-Valcárcel y Tejedor-Tejedor (2010) explican los problemas que se presentan al momento del uso e integración de las TIC en la enseñanza (p. 127):

- Ineficaz formación del profesorado para el uso de tecnología adecuada a las necesidades de los alumnos.

- Escaso tiempo disponible de los profesores para la colaboración entre ellos y el desarrollo de programas de tecnología integrada.

- Carencia de personal disponible para mantener los ordenadores y solucionar problemas técnicos y de aplicación didáctica (Coordinador de TIC)

- Falta de ordenadores y accesibilidad a Internet en todas las aulas (no sólo en laboratorios y aulas específicas).

Esta realidad también se manifiesta en la educación superior, las universidades alrededor del mundo se han valido de las TIC para desarrollar una educación que vaya más allá del campo universitario y acortar distancias en el acceso a la educación, por lo cual pasan por un proceso de virtualización que permite incluso acceder a estudios a través de medios virtuales o mejorar métodos pedagógicos.

El estudiante de educación superior está viviendo a plenitud la era de la tecnología, la cual participa en su formación como futuro profesional. Asimismo, los sistemas educativos del mundo se enfrentan al desafío de usar herramientas tecnológicas que otorguen a los estudiantes los conocimientos acordes a la era moderna.

Es tan relevante la inclusión de las herramientas tecnológicas en la educación que en varios países de Latinoamérica tales como Chile, Argentina, Brasil y Colombia se han implementado políticas educativas en las que se evidencia la aplicación masiva de las TIC en todas las instancias y niveles de enseñanza, cabe recalcar que los modelos educativos se circunscriben dentro de la realidad y contexto nacional del país que lo aplica.

En Ecuador, según estudios realizados por el Instituto Nacional de Estadísticas y Censos (INEC, 2019) acerca del uso de las TIC, el 36\% de los hogares ecuatorianos tuvieron acceso a internet, los usuarios más frecuentes de este recurso fueron los jóvenes de entre 16 y 24 años, rango al que pertenecen mayoritariamente estudiantes entre ellos los universitarios. Igualmente 8 de cada 10 jóvenes dentro de este mismo rango, usaron computadora y el $65.3 \%$ poseía teléfono celular durante ese año. La implementación de las TIC en la educación superior ha permitido el acceso a entornos virtuales de aprendizaje, espacios que permiten la interacción simultánea y concurrente entre estudiantes tales como: sitios web, aulas virtuales, blogs, etcétera, que otorgan recursos de aprendizaje en cualquier momento como transmisión de eventos e información, tutorías temáticas grabadas, ejercitadores, foros, videoconferencias, cursos online, entre otros.

La presente investigación tiene como objetivo determinar si la aplicación y uso de las TIC en el aula tiene una incidencia significativa en el rendimiento académico de los estudiantes que cursan la materia de Matemáticas Financieras en el periodo académico 2018 - 2019 ciclos I y II de la carrera de Administración de Empresas de la Universidad de Guayaquil, para esto se aplicó la Regla de Bayes o Teorema de Bayes para probar que la probabilidad de seleccionar un 
estudiante que usó TIC en el aula y que además tenga mejor rendimiento académico es mayor que un estudiante no usó TIC en el aula.

En el primer apartado se revisa la fundamentación literaria referente al uso y acceso de las TIC en sistemas educativos, así como su contribución a los aprendizajes y desempeños académicos. En el segundo capítulo se comparte la metodología que se utiliza en el estudio para luego, en el tercer capítulo describir los principales resultados de la investigación. Finalmente, se discuten los resultados con la evidencia disponible para luego ofrecer algunas conclusiones y recomendaciones.

\section{Revisión literaria}

La educación es un proceso en el que los individuos se sujetan a una socialización. Una persona al educarse, adquiere conocimientos; también aplica una concienciación cultural y conductual, es un hecho intencionado y que, en términos de calidad, persigue mejorar el rendimiento académico del estudiante.

En este sentido, Carrillo et al.(2010) indica que mejorar la calidad de la educación es prioridad en la mayoría de los países en vías de desarrollo y que la provisión y el uso de las TIC a las instituciones educativas pueden incrementar logros académicos en al menos dos maneras. Primero, la disponibilidad de las TIC en el salón de clases cambia sustancialmente el nivel de los componentes educativos que podrían afectar en los resultados de aprendizaje de los estudiantes. Segundo, la exposición a las TIC incrementaría las habilidades cognitivas de los estudiantes que permitiría un aprendizaje más rápido. En ciertos casos, la instrucción asistida con herramientas tecnológicas podría ser más significativo o relevante cuando la calidad del profesor se la considera corta.

En cualquier investigación de índole educativa la variable que con frecuencia se pretende analizar es el rendimiento académico, particularmente, en la enseñanza universitaria ya que representa uno de los temas más discutidos. Al respecto, González López (2004) expresa: «se trata de un concepto identificado como un constructo que se interpreta de distintas maneras en función del significado que tiene para las diferentes audiencias implicadas» (p. 44). Mientras que Forteza define al rendimiento académico como «la productividad del sujeto, el producto final de la aplicación de su esfuerzo, matizado por sus actividades, rasgos y la percepción más o menos correcta de los cometidos asignados» (apud García Jiménez et al., 2000).

Generalmente y desde el punto de vista práctico, al rendimiento académico se lo identifica con resultados, los mismos pueden distinguirse en dos categorías: inmediatos y diferidos (De Miguel Díaz y Arias Blanco, apud González López, 2004, p. 44). Se considera como rendimiento inmediato a aquellas calificaciones que obtienen los estudiantes a lo largo de su vida académica hasta conseguir la titulación correspondiente, en cambio; el rendimiento diferido se refiere a la aplicación de los conocimientos recibidos por los titulados en la vida profesional.

Uno de los temas más reiterativos dentro de la literatura docente en las últimas décadas es, sin duda, los factores que determinan el rendimiento académico de los estudiantes 
universitarios. En efecto, muchas investigaciones de este tipo se han realizado en distintos escenarios del ámbito universitario (Barahona U., 2014; Gomez D et al., 2011; Mushtaq y Nawaz Khan, 2012; Tomás J et al., 2014). Desde el contexto universitario, escenario donde se ha realizado la presente investigación, las contribuciones han sido importantes. De esta manera, los resultados de estas investigaciones explican que el rendimiento académico es el producto de la interacción de una serie de factores que inciden en el estudiante, concluyendo de esta forma que, es el resultado de la actividad docente que se ejerce en el alumno, aunque el rédito del aprendizaje no proviene del cien por ciento de la misma, sino que también de la predisposición que tenga el estudiante para aprender y del entorno en el que se desenvuelve.

La ingente literatura disponible, concluye que las tecnologías de la información y comunicación (TIC) es uno de los factores que inciden en el rendimiento académico de los estudiantes. Su incorporación a la educación ha sido un proceso con dificultades puesto que su implementación y uso ha generado cierta renuencia, muchas veces por parte del docente. Esta resistencia responde a que la formación de los profesores se realizó de forma tradicional, por ende no se encuentran familiarizados con las TIC y sus lenguajes (Sunkel, 2006, p. 44).

Dentro de la temática de TIC, se destacan estudios como el de Román (2014, p. 891) en el que analiza el acceso y uso de las TIC en el desempeño del estudiante y concluye que aquellos que tienen acceso a una computadora en casa reportan un mayor logro académico que los que no la poseen; además que existe una relación significativa entre el logro escolar y la frecuencia con la que el estudiante usa la computadora en el centro educativo. Esta investigación también registra un aporte desde la perspectiva docente, señala que si el profesor utiliza la computadora desde la casa el rendimiento académico del estudiante aumenta, contrario a lo que sucede si el docente usa la computadora en el centro de estudios o no, donde no parece tener ningún efecto.

Estudios como el de Alderete et al. (2017) realizado en España con un Modelo de Ecuaciones Estructurales (SEM) concluye que el acceso a las TIC en el hogar tienen un efecto positivo y estadísticamente significativo sobre el rendimiento académico, en contraste al acceso y uso de las TIC en el centro educativo, que tienen una incidencia negativa y estadísticamente significativa en los logros académicos. El mismo estudio, realizado en Argentina por Formichella et al., (2015, p. 369) concluye que existen diferencias significativas entre grupos de estudiantes que acceden a las TIC desde sus hogares con aquellos que acceden desde el centro de estudio, es decir, el acceso y uso potencial de las TIC desde el hogar inciden de manera positiva y estadísticamente significativa en el rendimiento del estudiante.

Por su parte, Castro Aristizabal et al., (2012) realizó una investigación en Colombia, cuyos resultados difieren de otras investigaciones expuestas. Este estudio concluye que el acceso a las TIC tanto en el hogar como en el centro educativo tienen una incidencia positiva y estadísticamente significativa en el rendimiento del estudiante, expone además que, dentro del alcance de las tecnologías en el aula, la mayor contribución académica la proporcionó el uso de computadoras mientras que el uso de internet y sus herramientas en el hogar, tuvieron un mayor peso académico.

Si el objetivo es alcanzar un mejor aprendizaje y rendimiento académico, no es suficiente poner a disposición estas tecnologías, es necesario una sutil combinación de hardware (provisión 
de computadoras y un laboratorio), software (plataformas de aprendizajes virtuales) y desde luego, un docente capacitado se puede alcanzar importantes logros académicos para el estudiante. Así lo muestra Carrillo et al. (2010) en su investigación, en donde los resultados muestran que una adecuada interacción de estos tres elementos arroja diferencias significativas en grupos de control y tratamiento.

De acuerdo con la revisión teórica, se puede concluir que las tecnologías de la información y comunicación influyen en el rendimiento académico del estudiante. En algunas investigaciones, los resultados han demostrado que estas herramientas tienen una incidencia estadística más significativa dentro del hogar y no tan relevante dentro del centro de estudio.

\section{Metodología}

Esta investigación de carácter empírico se apoya en una metodología descriptiva de corte transversal en la que se muestran las diferencias que existe en el rendimiento académico entre dos grupos de estudiantes, definiendo la variable "rendimiento académico" como la calificación que obtiene el estudiante en la asignatura al finalizar el semestre. La escala de calificación es sobre diez puntos.

Para este estudio se ha considerado a los estudiantes de la materia de Matemáticas Financieras de segundo semestre de la carrera de Administración de Empresas de la Universidad de Guayaquil en los periodos académicos 2018 - 2019 ciclos I y II. Ciento cuarenta y cuatro estudiantes recibieron la asignatura de la forma tradicional, es decir el docente explica los contenidos con texto físico, pizarra acrílica, marcador y borrador mientras que ciento setenta y uno estudiantes recibieron la asignatura mencionada utilizando herramientas tecnológicas en el aula: computadora, proyector, dispositivas y textos electrónicos.

En esta investigación se plantean dos hipótesis:

H1: El promedio final de la asignatura de Matemáticas Financieras para los estudiantes que no utilizaron TIC es menor al de los estudiantes que utilizaron TIC.

H2: La probabilidad de seleccionar un estudiante que tenga una calificación igual o mayor a 7.5 sobre 10 dado que recibió sus clases con TIC está por encima del cincuenta por ciento.

Para probar la primera hipótesis, se compara la calificación final de los estudiantes que no usaron ninguna herramienta tecnológica y los que estudiantes que usaron TIC en el aula mediante una descripción estadística de las muestras independientes. En tanto que, para la segunda hipótesis se utiliza una aplicación del Teorema de Bayes o la Regla de Bayes.

Según (Walpole et al., 2013) la estadística bayesiana es un conjunto de herramientas que se utiliza en un tipo especial de inferencia estadística que se aplica en el análisis de datos experimentales. El teorema de Bayes es una de las normas más importantes de la teoría de probabilidad ya que expresa la probabilidad de carácter condicional de un evento aleatorio dad cierta información de antemano sobre el suceso. 
Es decir, calcula la probabilidad de que ocurra un evento A condicionado por la información del evento B. De esta manera se determina la probabilidad de las causas a partir de los efectos que han podido ser observados.

La regla de Bayes indica que si los eventos $B_{1}, B_{2}, \ldots, B_{k}$ constituyen una partición del espacio muestral $S$, donde $P\left(B_{i}\right) \neq 0$ para $i=1,2, \ldots, k$, entonces para cualquier evento $A$ en $S$, tal que $P(A) \neq 0$

$$
P\left(B_{r} \backslash A\right)=\frac{P\left(B_{r} \cap A\right)}{\sum_{i=1}^{k} P\left(B_{i} \cap A\right)}=\frac{P\left(B_{r}\right) P\left(A \backslash B_{r}\right)}{\sum_{i=1}^{k} P\left(B_{i}\right) P\left(A \backslash B_{i}\right)} \quad \text { Ecuación 1 }
$$

para $r=1,2, \ldots, k$

El numerador de la ecuación 1 es la probabilidad condicionada y el denominador es la probabilidad total.

\section{Resultados}

En primera instancia, se realizó un análisis descriptivo con el fin de ver el comportamiento de cada una de las muestras, con el soporte de un paquete estadístico se observan los siguientes resultados:

\section{Resultados sin herramientas tecnológicas en el aula}

Como se puede observar en la Tabla 1. la media de calificaciones de los estudiantes que recibieron clases sin TIC es de 6.85 puntos. El 50\% de los estudiantes de este curso tienen calificaciones por debajo de 7.30 mientras que la calificación que se repitió en mayor cantidad entre los estudiantes fue de 7 puntos. Tal como se observa, la variabilidad de los datos es 1.82 puntos con respecto a la calificación promedio del curso; además, se puede observar en la Gráfica 1. que la mayor cantidad de datos se encuentran a la izquierda de la media debido a que el sesgo es negativo, esto significa que la mayoría de las calificaciones de los estudiantes están por debajo de la media. Este grupo de estudiantes tiene una calificación mínima de 1.54 puntos y una calificación máxima de 9.48 puntos.

\section{Tabla 1.}

Calificación Final sin TIC

\begin{tabular}{cc}
\hline Estadístico & Valor \\
\hline Media & 6.85222222 \\
Error típico & 0.15237359 \\
Mediana & 7.305 \\
Moda & 7 \\
Desviación estándar & 1.82848305 \\
Varianza de la muestra & 3.34335027 \\
Curtosis & 1.06977137 \\
Coeficiente de asimetría & -1.4303622
\end{tabular}




\begin{tabular}{lcc}
\cline { 2 - 3 } & Estadístico & Valor \\
\cline { 2 - 3 } & Rango & 7.94 \\
Mínimo & 1.54 \\
Fuente: Elaboración propia & Máximo & 9.48 \\
& Suma & 986.72 \\
\cline { 2 - 3 } & Cuenta & 144 \\
\hline
\end{tabular}

\section{Gráfica 1.}

Calificación Final sin TIC

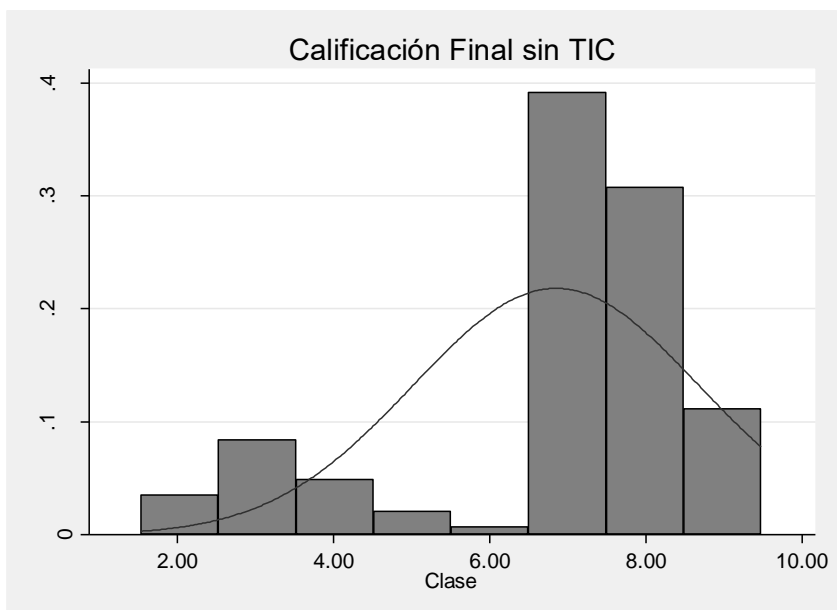

Fuente: Elaboración propia

\section{Resultados con herramientas tecnológicas en el aula}

La Tabla 2 muestra que la calificación media de los estudiantes que recibieron clases con herramientas tecnológicas es de 7.68 puntos, el 50\% de los estudiantes tienen calificaciones menores de 8 y la calificación que más se repitió fue de 7 puntos. La variabilidad de las calificaciones de los estudiantes que recibieron sus clases con TIC es de 1.07 con respecto a la calificación promedio del curso, esto indica que existe una alta concentración de observaciones alrededor de la media. Adicionalmente, se puede observar en la Gráfica 2 que la mayor cantidad de datos se encuentran a la izquierda de la media debido al que el sesgo es negativo, es decir la mayoría de los estudiantes obtuvieron calificaciones por debajo de la media. La nota mínima que se presentó en este grupo de estudiantes fue de 3 mientras que la máxima fue de 10 puntos. 


\section{Gráfica 2.}

Calificación Final con TIC

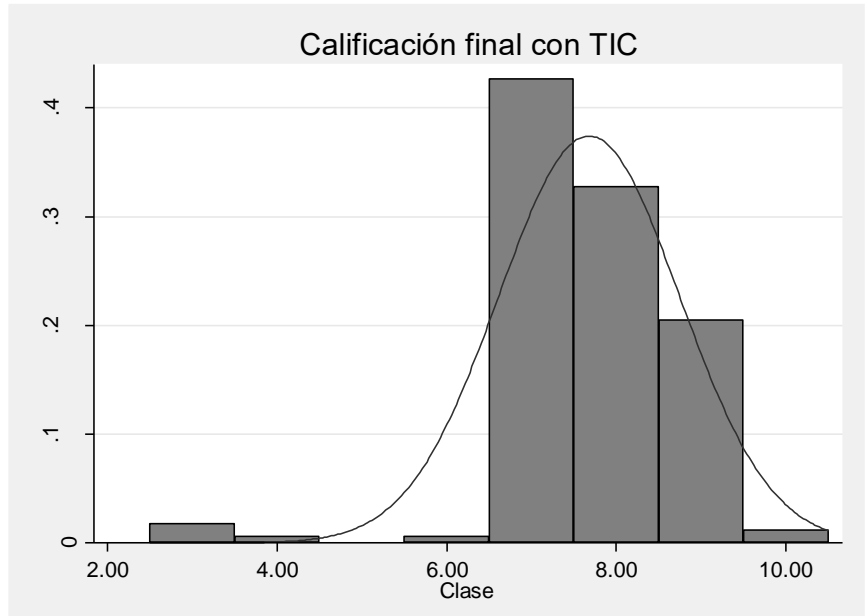

Fuente: Elaboración propia

Tabla 2.

Calificación Final con TIC

Fuente: Elaboración propia

\begin{tabular}{lr}
\hline \multicolumn{1}{c}{ Estadístico } & \multicolumn{1}{c}{ Valor } \\
\hline & 7.67836257 \\
Media & 0.08154636 \\
Error típico & 8 \\
Mediana & 7 \\
Moda & 1.06635702 \\
Desviación estándar & 1.1371173 \\
Varianza de la muestra & 5.47199171 \\
Curtosis & -1.35860159 \\
Coeficiente de asimetría & 7 \\
Rango & 3 \\
Mínimo & 10 \\
Máximo & 1313 \\
Suma & 171 \\
Cuenta & \\
\hline
\end{tabular}

Con estos resultados se puede concluir que los estudiantes que reciben clases con TIC tienen un rendimiento académico mayor que aquellos que reciben clases sin herramientas tecnológicas. Dado que el valor del estadístico t es mayor que el valor crítico, existe suficiente evidencia estadística para rechazar la hipótesis nula y aceptar la hipótesis alternativa.

Para comprobar la segunda hipótesis de esta investigación procedemos a realizar un diagrama de árbol. 


\section{Gráfica 3.}

Árbol de decisión

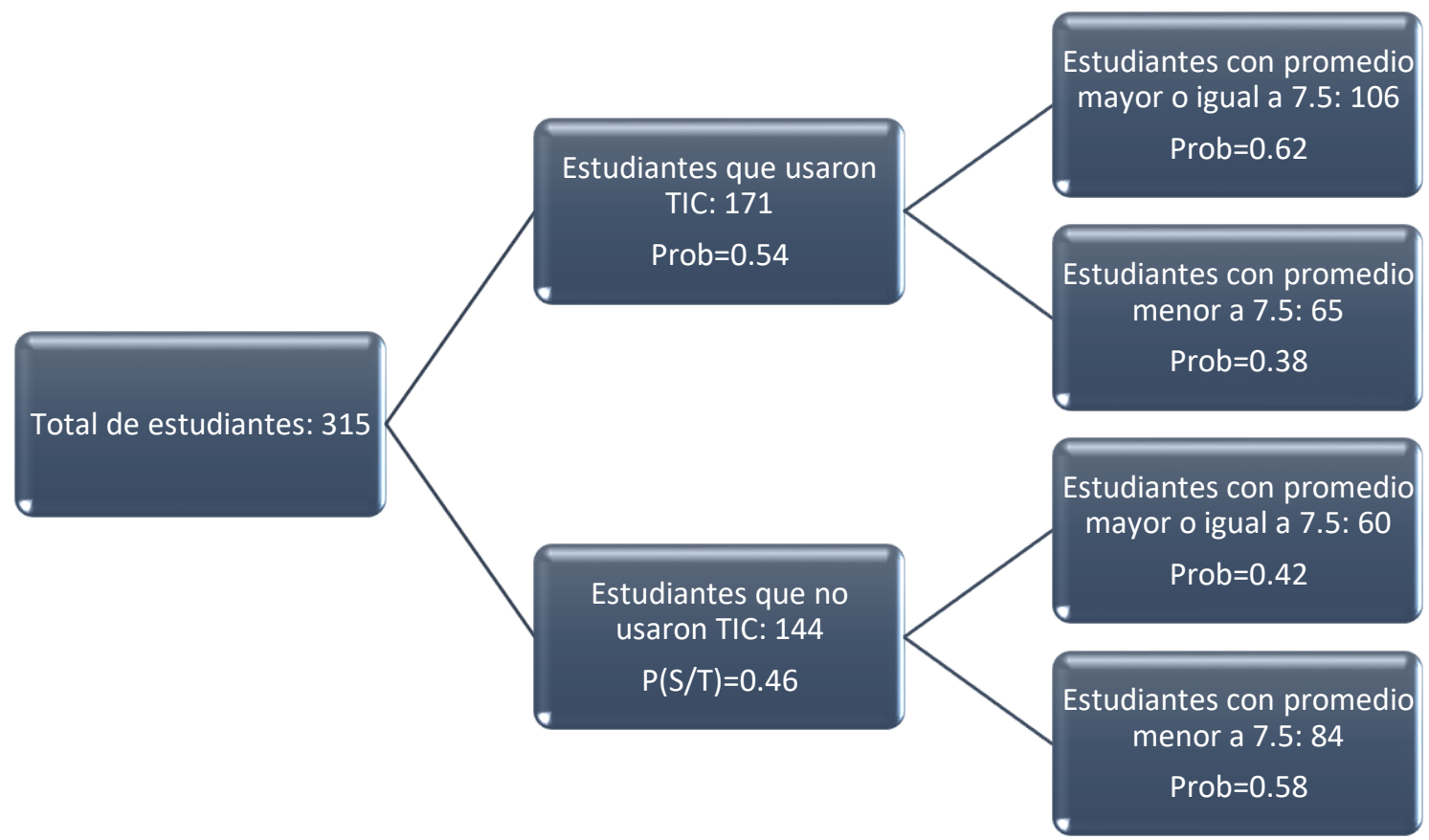

Fuente: Elaboración propia

Lind et al. (2014) sostiene que «el diagrama de árbol es una gráfica útil para organizar cálculos que implican varias etapas». Las ramas de este árbol de decisión constituyen una etapa del problema que se ponderan por medio de probabilidades.

La Gráfica 3 muestra la construcción de un diagrama de árbol para una mejor aplicación de la Ecuación 1.

$$
\begin{gathered}
P(\text { con TIC \promedio }>7.5)=\frac{(0.54)(0.62)}{(0.54)(0.62)+(0.46)(0.42)} \\
P(\text { con TIC \promedio }>7.5)=0.6341 \equiv 63.41 \%
\end{gathered}
$$

\section{Conclusiones}

La implementación y el uso de las TIC es un tema que por muchas décadas ha estado dentro de los temas principales de investigaciones educativas. Es innegable el rol que tienen las tecnologías de información y comunicación y su incidencia en la formación académica. El presente artículo analiza la importancia que tiene la aplicación de las TIC dentro del aula de clase y como éstas influyen en las calificaciones finales de los estudiantes que tomaron la materia de 
Matemáticas Financieras en la carrera de Administración de Empresas de la Universidad de Guayaquil durante el periodo 2018 -2019 ciclos I y II.

Con respecto a los resultados, mediante un análisis descriptivo se comprobó que los estudiantes que reciben sus clases con la aplicación de herramientas tecnológicas tienden a tener un mejor rendimiento académico que aquellos estudiantes que no recibieron sus clases con TIC.

También se analizó la aplicación de la Regla de Bayes para estimar (predecir) la probabilidad de que un estudiante escogido aleatoriamente tenga un mejor rendimiento académico y para esta muestra no probabilística resultó que existe un $63.41 \%$ de posibilidad con lo que se demuestra que la aplicación de las TIC en el aula mejora la calificación promedio del estudiante.

El estudio de las TIC en la educación se encuentra en una etapa de crecimiento. Con esta investigación se pretende incentivar a instituciones educativas, docentes y a estudiantes a usar herramientas tecnológicas dentro del aula para que, de esta manera, los estudiantes proactivos se puedan integrar mejor a nuevas metodologías.

\section{Bibliografía}

Alderete, M. V., Di Meglio, G., \& Formichella, M. M. (2017). Acceso a las TIC y rendimiento educativo: ¿una relación potenciada por su uso? Un análisis para España. Revista de Educacion, 2017(377), 54-79. https://doi.org/10.4438/1988-592X-RE-2017-377-353

Alderete, M. V., \& Formichella, M. M. (2016). Efecto de las TIC en el rendimiento educativo: el Programa Conectar Igualdad en la Argentina. Revista Cepal, 119, 89-99. http://eds.b.ebscohost.com/eds/pdfviewer/pdfviewer?vid=0\&sid=2da398a9-9617-44e083f9-4dd679bea95a\%40sessionmgr120

Barahona U., P. (2014). Factores determinantes del rendimiento académico de los estudiantes de la Universidad de Atacama. Estudios Pedagogicos, 40(1), 25-39. http://www.redalyc.org/articulo.oa?id=173531772002

Carrillo, P., Onofa, M., \& Ponce, J. (2010). Information Technology and Student Achievement : Evidence from a Randomized Experiment in Ecuador Information Technology and Student Achievement : Evidence from a Randomized Experiment. In IBD Working Paper (IDB-WP-223; Issue December). http://www.iadb.org

Castro Aristizabal, G., Castillo Caicedo, M., \& Escandón, D. M. (2012). Las Tecnologías de la Información y Comunicación como determinante en el rendimiento académico escolar, Colombia 2006-2009. In E. Pacheco Vieiria (Ed.), Investigaciones de economía de la educación (7th ed., pp. 889-904). Investigaciones de Economía de la Educación.

Formichella, M. M., Alderete, M. V., \& Di Meglio, G. (2015). El acceso a las TIC en el hogar como determinante del rendimiento educativo en el nivel medio: un análisis para Argentina. In M. Rahona López \& J. Graves (Eds.), Investigaciones de la Economía de la Educación (1st ed., pp. 357-374). Asociación de Economía de la Educación.

García-Valcárcel, A., \& Tejedor Tejedor, F. (2010). Evaluación de procesos de innovación escolar basados en el uso de las TIC desarrollados en la comunidad de castilla y león. Revista de Educacion, 352(1), 125-147.

García Jiménez, M. V., Alvarado Izquierdo, J. M., \& Jiménez Blanco, A. (2000). La predicción 
del rendimiento académico : regresión lineal versus regresión logística. Psicothema, 12(2), 248-252.

Gomez D, Oviedo R, \& Martinez E. (2011). Factores que influyen en el rendimiento academico del estudiante universitario. Educacion y Humanidades [revista en Internet] 2011 [acceso 12 de octubre de 2018]; 5(2): 90-97. $V(2)$. http://tecnociencia.uach.mx/numeros/v5n2/data/Factores_que_influyen_en_el_rendimie nto_academico_del_estudiante_universitario.pdf

González López, I. (2004). Realización de un análisis discriminante explicativo del rendimiento académico en la universidad. Revista de Investigación Educativa, 22(1), 43-59.

INEC. (2019). Encuesta de Seguimiento al Plan Nacional de Desarrollo.

Lind, D. A., Marchal, W. G., Wathen, S. A., Iván, J., Sánchez -Farley, J., Rojas, S., Julieth, R.-H., Galvis, O., Iván Jiménez Sánchez, J., Sary, F., Restrepo, R., Julieth, H., Scherger, V. P., Arbelaez R, M. A., Zulet J, L. A., Velazco M, A., Rosales R, M. F., Dominguez G, C., Economicas, D. E. C., ... Analisis, E. N. E. L. (2014). Estadística Aplicada a los Negocios Y La Economía. In Ciencia y Sociedad: Vol. XVI (Issue 4). https://doi.org/10.22206/cys.2011.v36i4.pp590-627

Mushtaq, I., \& Nawaz Khan, S. (2012). Factors Affecting Students' Academic Performance. Global Journal of Management and Business Research, 12(9), 2648-2652. https://doi.org/10.1109/nafips.2001.943641

Ricardo Barreto, C., Iriarte Diazgranados, F., \& Hug, E. S. (2017). Las TIC en educación superior : experiencias de innovación (Z. Sotomayor O. (ed.); 23rd ed.). universidad del Norte. http://rd.unir.net/sisi/research/resultados/15119077649789587418552 eLas TIC en la educacion superior.pdf

Román, M., \& Murillo, F. J. (2014). Disponibilidad y uso de TIC en escuelas latinoamericanas : incidencia en el rendimiento escolar. Educação e Pesquisa, 40(4), 879-895. https://doi.org/http://dx.doi.org/10.1590/s1517-97022014121528

Romaní, J. C. C. (2011). El concepto de tecnologías de la información. Benchmarking sobre las definiciones de las TIC en la sociedad del conocimiento. Zer - Revista de Estudios de Comunicación, 14(27). http://www.ehu.eus/ojs/index.php/Zer/article/view/2636

Sánchez, A; Boix, Josep; Jurado, P. (2009). LA SOCIEDAD DEL CONOCIMIENTO Y LAS TICS: UNA INMEJORABLE OPORTUNIDAD PARA EL CAMBIO DOCENTE. Revista de Medios y Educación, 34, 179-204. https://www.redalyc.org/articulo.oa?id=368/36812036013

Sunkel, G. (2006). Las Tecnologías de la información y comunicación (TIC) en la educación en América Latina. Una exploración de indicadores.

Tomás-Miquel, J.-V., Expósito-Langa, M., \& Sempere-Castello, S. (2014). Determinantes del rendimiento académico en los estudiantes de grado. Un estudio en administración y dirección de empresas. Revista de Investigación Educativa, 32(2), 379-392. https://doi.org/10.6018/rie.32.2.177581

Walpole, ronald E., Myers, R. H., Myers, S. L., \& Ye, K. (2013). Probabilidad y estadística para ingeniería y ciencias (Pearson (ed.); Novena Edi, Vol. 369, Issue 1). 